\title{
Aplicación de la Teoría del Cambio para la Gestión de Proyectos Públicos
}

\author{
Restrepo Carvajal, Carlos Alberto ${ }^{1\left(^{*}\right)}$; \& Gómez Arenas, Natalia Andrea ${ }^{2}$
}

${ }^{1}$ Universidad Centro Panamericano de Estudios Superiores UNICEPES, Morelia, México.

${ }^{2}$ Tecnológico de Antioquia, Institución Universitaria, TdeA; Medellín, Colombia.

\section{RESUMEN}

La Gestión de Proyectos Públicos requiere de una mirada integradora a partir de la concepción del ciclo de vida y de la cadena de valor de la organización, para la convergencia de las necesidades de la población y la medición del efecto o impacto del proyecto. Para esta imbricación, se propone la aplicación de la Teoría del Cambio, la cual permite el desarrollo de la cadena causal del constructo de análisis, considerando las necesidades de la población, los recursos, los entregables, los resultados intermedios (victorias tempranas) y finales. Asimismo, en corolario con las relaciones causales, la Teoría favorece el monitoreo de los resultados a través de las métricas soportadas en los supuestos y restricciones del proyecto o programa. En consonancia con lo anterior, la gestión de proyectos públicos se beneficia de la inserción de esta Teoría en la estructuración de programas, portafolios y proyectos, debido a que se establece una relación palmaria entre los requerimientos de desarrollo y las potencialidades del territorio. Se presentan los resultados de la aplicación de la Teoría del Cambio asociados a la estructuración de un modelo educativo, el cual parte de las necesidades de los estudiantes.

Palabras clave: Gestión de Proyectos Públicos; Teoría del Cambio; Educación; Estructuración de Proyectos

\section{Application of the Theory of Change for the Management of Public Projects}

\section{ABSTRACT}

The Management of Public Projects requires an integrative look from the conception of the life cycle and the value chain of the organization, for the convergence of the needs of the population and the measurement of the effect or impact of the project. For this overlapping, the application of the Theory of Change is proposed, which allows the development of the causal chain of the analysis construct, considering the needs of the population, resources, deliverables, intermediate results (early victories) and final. Likewise, as a corollary to the causal relationships, the Theory favors the monitoring of the results through the metrics supported by the assumptions and restrictions of the project. In line with the above, the management of public projects benefits from the insertion of this Theory in the structuring of programs, portfolios and projects, because a clear relationship is established between development requirements and the potential of the territory. The results of the application of the Theory of Change associated with the structuring of an educational model are presented, which is based on the needs of the students.

Keywords: Public Project Management; Education; Theory of Change; Project Structuring 


\section{INTRODUCCIÓN}

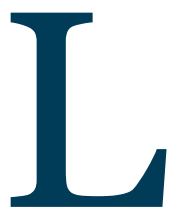

La Teoría del Cambio es una técnica de análisis que permite la comprensión de la cadena de valor de un proyecto o programa. En este sentido, fomenta el logro de la implementación de una política pública materializada a través de la relación de necesidades de la población y el impacto asociado a la alteración de las condiciones del entorno.

En la gestión pública es fundamental la medición de impacto de la implementación de una política a través de proyectos, lo cual se establece a partir de la diferencia entre el factual y el contrafactual. En la Figura 1, se relaciona la definición de impacto. En este mismo sentido, en este análisis se involucra la cadena de valor y los ciclos de vida del proyecto y del gerenciamiento, para potenciar la comprensión del efecto esperado en la comunidad, a partir de la interacción con los resultados finales.

Rogers, P. (2014) define el Factual, como el resultado que obtienen los participantes del proyecto o programa después de participar en éste. Mientras que el Contrafactual se establece como el resultado que esos mismos participantes hubieran obtenido en el periodo del proyecto, en el caso hipotético de no haber participado en el programa.

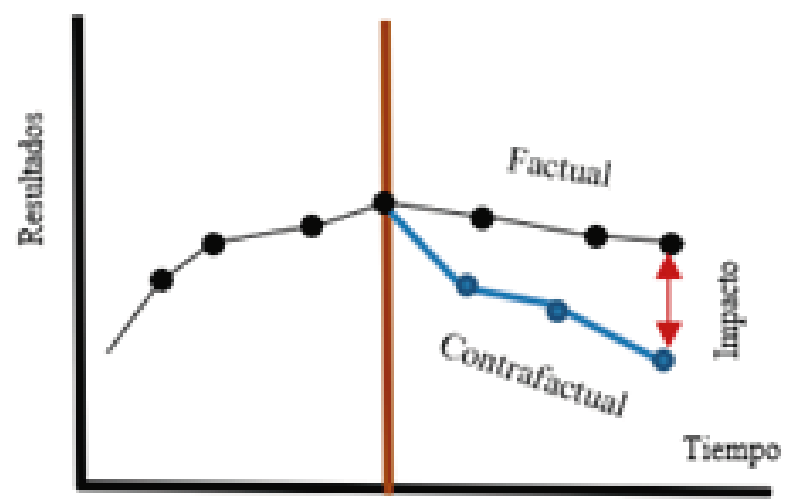

Figura 1. Definición de Impacto.

Fuente: Elaboración propia.

\section{MARCO TEÓRICO}

En consonancia con lo anterior, en la evaluación de proyectos es usual la aplicación de la Matriz de Vester como herramienta de priorización y causalidad; además, facilita el análisis de las variables asociadas al factual (Restrepo, C. \& Cuadros, A (2013)). En este orden de ideas, a partir del diagrama causal de esta matriz se definen las variables independientes, asociativas, autónomas y dependientes. Sumper (2010) encuentra que la evaluación de impacto consiste en la construcción de una matriz, donde mediante índices de ponderación y escalas de valoración, se definen los impactos más fuertes, y sobre los cuales se debe actuar prioritariamente.

Para el desarrollo de la Teoría del Cambio se requiere que las expectativas y necesidades de los grupos de valor estén claramente identificadas. A partir de éstas y de los resultados finales e impactos, es plausible definir las variables relevantes de la Teoría; y la satisfacción de estos grupos, a través de la siguiente relación:

Satisfacción $=$ Percepciones - Expectativas

Es de anotar, que, las percepciones se miden al final de un proceso o etapa del ciclo de vida del proyecto; mientras que las expectativas se obtienen al inicio.

La Figura 2 presenta la Cadena de Valor, la cual permite un discernimiento de la relación de los grupos de valor, necesidades y productos. El reto está en una definición diáfana de las expectativas de estos grupos, para establecer los niveles de satisfacción una vez el programa esté finalizado.

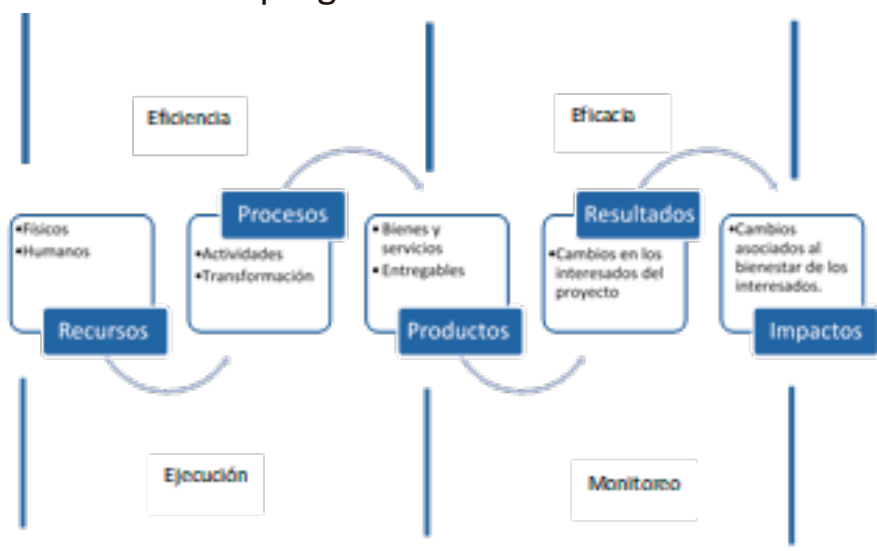

Figura 2. Cadena de Valor.

Fuente: Elaboración propia. 
El PMI (2017), en su Guía de Proyectos, sugiere que, para cada etapa del ciclo de vida del proyecto, se relacione el ciclo de vida del gerenciamiento del proyecto, lo cual favorece el monitoreo a las métricas y el logro de los resultados. En la Figura 3 se presentan las etapas de inicio, planeación, implementación, monitoreo y control y cierre.

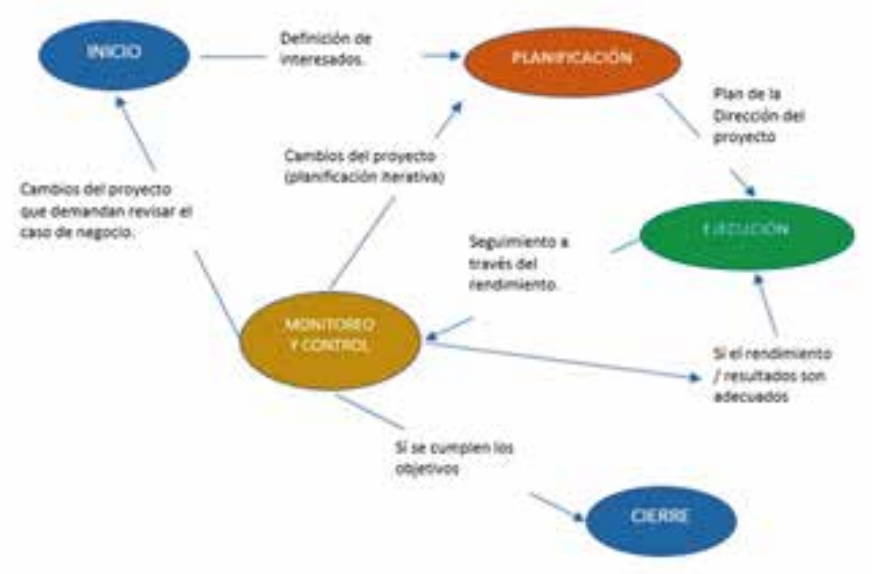

Figura 3. Ciclo de Vida del Gerenciamiento de Proyectos. Fuente: Elaboración propia.

De conformidad con la medición del impacto del proyecto, en función del cambio de la calidad de vida de la muestra de la población, es fundamental que la Entidad Pública determine el éxito del proyecto.

Istrate et al (2014) definen que para alcanzar el éxito de un proyecto es necesario responder los siguientes interrogantes:

- ¿CóMO? - Lo que justifica una función a través de funciones de grado inferior y hace hincapié en la construcción de árbol funciones. Se asocia con el método y herramientas.

- ¿PARA QUÉ? - Que justifica la contribución de las funciones de un grado inferior para los principales en una función compleja de la función de un grado inferior.

- ¿CUÁNDO? - El tiempo de cuando se alcanza la disyunción, los momentos en los que la mayoría de las funciones se deben satisfacer simultáneamente.
Lo anterior conlleva a hacer una adecuada gestión de los proyectos, definiendo objetivos claros y una optimización de recursos que servirá para dar éxito a los mismos. Si al inicio de cada proyecto se definen estos tres puntos claramente el proyecto saldrá bien. De acuerdo con lo anterior, se presenta en la Figura 4, la conceptualización de éxito.

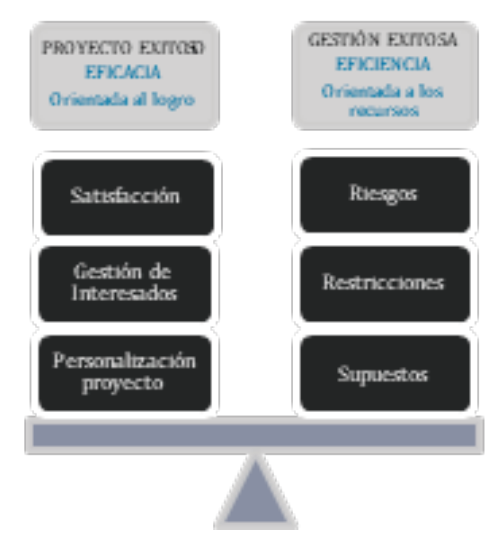

Figura 4. Medición de éxito en proyectos públicos. Fuente: Elaboración propia..

\section{MARCO TEÓRICO}

Rogers, P. (2014) describe la Teoría del Cambio como un conjunto de actividades encaminadas a generar resultados intermedios y finales que contribuyen al logro de los efectos de la política pública, gestionada mediante programas y proyectos. El modelo teórico de esta teoría se ilustra en la Figura 5. De acuerdo con este autor, es posible desarrollar una teoría del cambio bajo las siguientes premisas:

- Cuando los objetivos y las actividades del programa o proyecto pueden identificarse y planificarse.

- Cuando el programa se ajusta a las necesidades y decisiones de los grupos de valor.

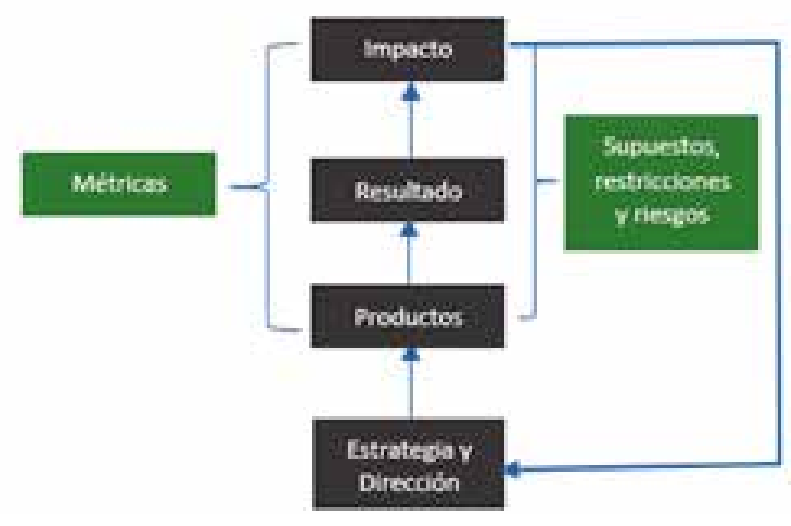

Figura 5. Modelo Teoría del Cambio.

Fuente: Adaptado de Rogers, P. (2014) 
Amundsen, C., \& D’Amico, L. (2019) establecen que la Teoría del Cambio examina los vínculos entre el diseño de un programa y el cambio esperado en los participantes. Además, con base en la interacción de éstos y del programa se definen las fuentes de evidencia apropiadas para demostrar las metas de la política pública que se desea implementar.

Estos autores presentan cuatro (4) aspectos a considerar para la implementación de la Teoría del Cambio:

-Articulación del propósito general del programa o proyecto con la política pública.

-Integración y diseño del propósito del programa con los resultados.

- Identificación de fuentes de evidencia que permitan comprender si el vínculo entre el propósito, el diseño y los resultados es palmario.

- Explicación de cómo la Teoría del Cambio se describe a través de la evidencia.

Theoryofchange.org define la Teoría del Cambio como una descripción e ilustración integral de cómo y por qué se espera que tome lugar un cambio deseado en un contexto definido. Esta organización propone inicialmente identificar las metas deseadas a largo plazo y luego a partir de ellas, establecer las condiciones (resultados) que deben estar en su lugar ( $y$ cómo se relacionan entre sí causalmente) para que las metas se materialicen. Weitzman, B.C. (2002) asevera que la definición de esta organización inicia con el trazado del proceso de logro de las metas con los grupos de valor. La tarea inicial es explicitar la Teoría del Cambio, considerando los supuestos y riesgos de la implementación del programa.

La Teoría del Cambio tiene como propósito abordar los vínculos entre los objetivos, las estrategias, los resultados y las suposiciones que respaldan la misión y la visión de un programa. Esta Teoría inició al tratar de comprender el funcionamiento de un programa que responde a una política públi$\mathrm{ca}$, explorando supuestos subyacentes sobre procesos de cambio y creencias de los territorios (Weiss, C.H., 1997).
De acuerdo con Stachowiak, S. (2013), la Teoría del Cambio es una herramienta de gestión que se utiliza para la planificación formal, involucrando los procesos, supuestos y restricciones para el desarrollo del programa o proyecto. Por lo anterior, esta Teoría se convierte en una hoja de ruta para ir de aquí para allá.

Cada vez es más frecuente el uso de la Teoría del Cambio en la implementación de políticas públicas, lo cual permite la interpretación de una necesidad social desde un enfoque integrador y causal. Este nuevo paradigma pone a la Teoría del Cambio como una herramienta ideal para planificar y evaluar la efectividad de las intervenciones de desarrollo social. (Arensman et al, 2018). Además, la Teoría del Cambio se refiere al modelo rector específico de los responsables de desarrollar, dirigir e implementar el programa. En este sentido, los evaluadores involucran al personal del programa para hacer explícitas la Teoría del Cambio y los supuestos implícitos en los programas de desarrollo público (DuBow, W. M., \& Litzler, E., 2019).

\section{DESARROLLO TEORÍA DEL CAMBIO}

El estudio se realiza mediante el enfoque estudio de caso y el paradigma crítico social. En el enfoque crítico social, la participación de actores, se desarrolla en cuatro fases: planificar, actuar, observar y desarrollar. Por su parte, el método estudio de caso se desarrolla considerando los datos de satisfacción de estudiantes de pregrado y posgrado de Instituciones de Educación Superior en Medellín. La interacción con los estudiantes se realiza mediante grupos focales y talleres facilitados. En la Figura 6 se presenta el esquema de la Teoría del Cambio para el estudio.

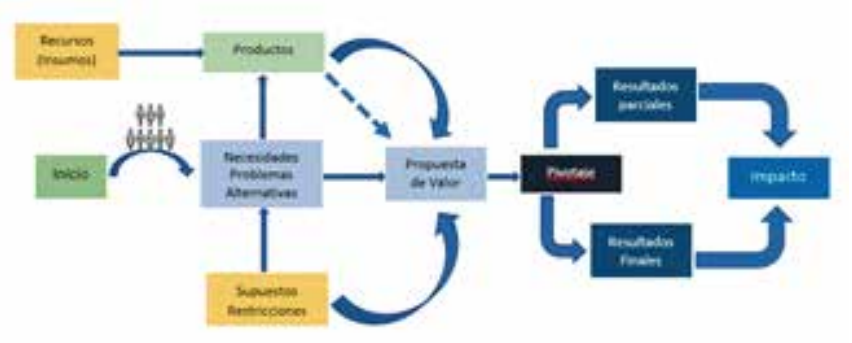

Figura 6. Modelo Teoría del Cambio.

Fuente: Elaboración propia 
El inicio del desarrollo del modelo de la Teoría del Cambio se elabora a partir de la definición de las necesidades y problemas de los participantes. Es plausible encontrar que la población esté intentando por sus medios subsanar o cubrir parcialmente sus necesidades. Para el diagnóstico de los participantes se elabora la Figura 7.

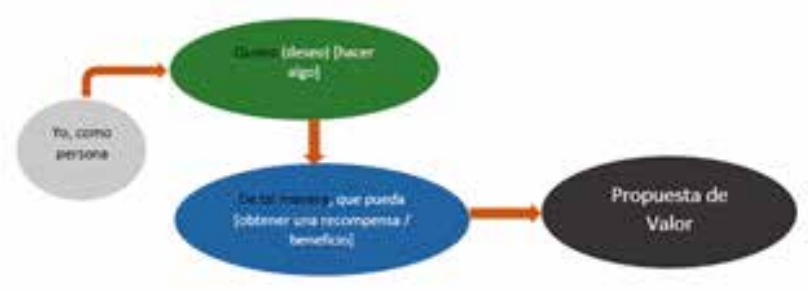

Figura 7. Modelo de gestión de necesidades.

Fuente: Elaboración propia

La propuesta de valor debe generar mayores beneficios que las alternativas actuales que la población (participantes) ejecuta. Para este propósito, se usa el Lean Canvas, como modelo de relacionamiento de la cadena de valor. En la Figura 8 se presenta el esquema del modelo.

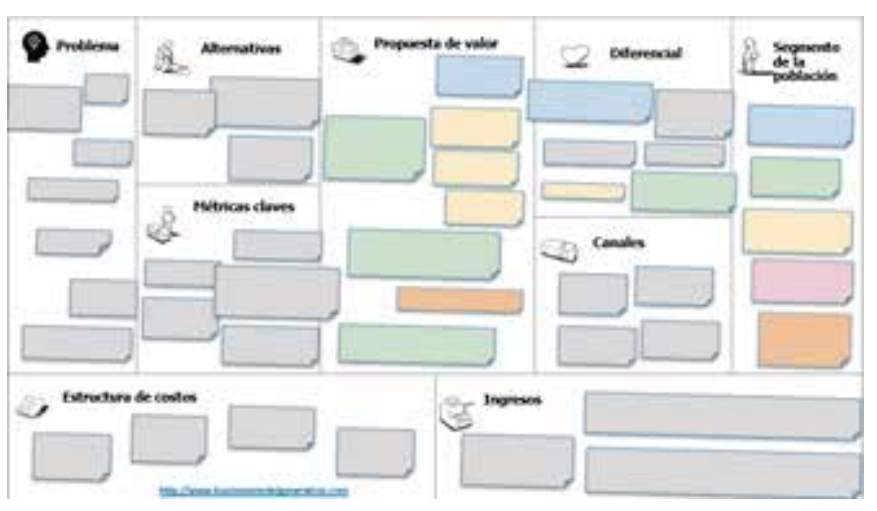

Figura 8. Lean Canvas.

Fuente: Adaptado de "

Entre la propuesta de valor y el impacto se encuentra un elemento denominado punto de pivote, el cual confronta que los resultados intermedios y finales subsanen las necesidades de la población. Sí las necesidades no se satisfacen es necesario iterar las necesidades, supuestos y productos.

En la Tabla 1 se presenta el esquema de trazabilidad del balanceo de las necesidades de los participantes. Es de anotar, que, este instrumente es dinámico.
Tabla 1. Registro de necesidades. Fuente: Elaboración propia.

\begin{tabular}{|c|c|c|c|c|c|c|}
\hline Interesado & Nesesidad & Aiternativa & Requerimlento & Deveripxión & $\begin{array}{l}\text { Criterios de } \\
\text { aceptarbon }\end{array}$ & Priceridad \\
\hline 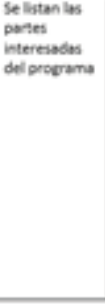 & $\begin{array}{l}\text { Las } \\
\text { necenidades } \\
\text { se mocien } \\
\text { con las } \\
\text { expectabivas } \\
\text { de la } \\
\text { poblasion }\end{array}$ & 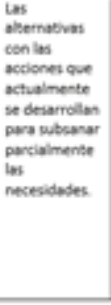 & 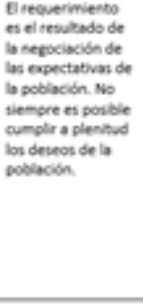 & 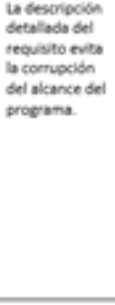 & 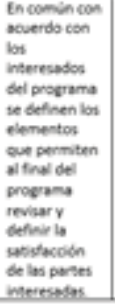 & 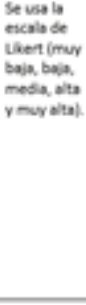 \\
\hline
\end{tabular}

En corolario con lo anterior, en la Figura 9 se presenta la estructura del modelo de Teoría del Cambio desarrollado para el estudio. Este modelo se construye a partir de la concepción del análisis de cadena de valor y ciclo de vida del gerenciamiento del proyecto. Rogers, P. (2014) propone que cada etapa del modelo debe incluir la participación de los evaluadores externos del programa.

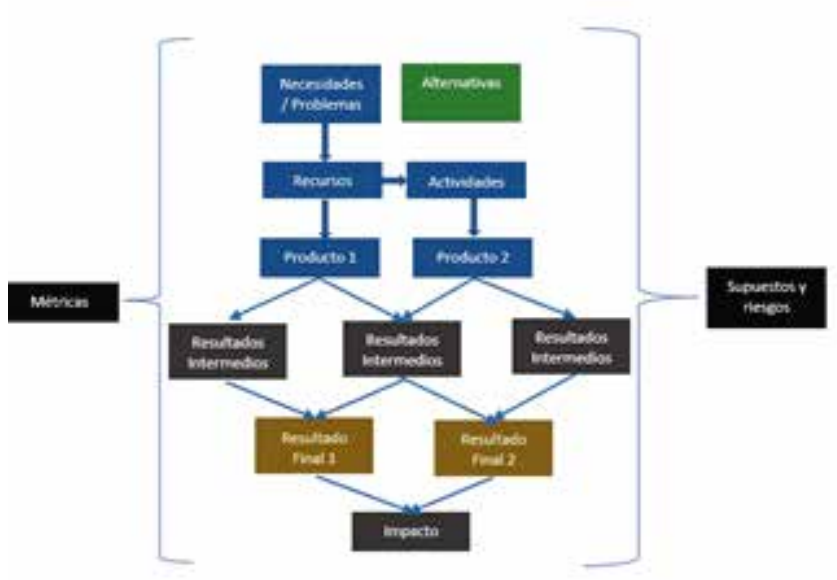

Figura 9. Modelo Integrado de Teoría del Cambio. Fuente: Elaboración propia

En la Figura 10 se ilustra la relación entre la meta del programa, los interesados, los impactos y los entregables (resultados). Esta estructura es una herramienta de comunicación con los grupos de valor, y se elabora en dos momentos, al inicio del programa, y al final de cada etapa definida para el proyecto.

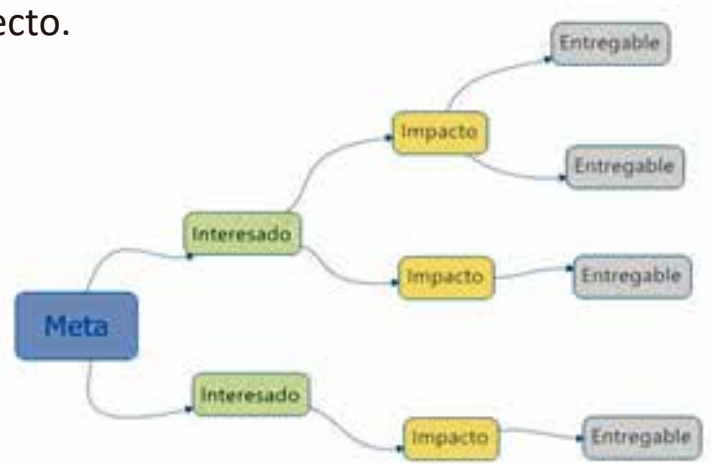

Figura 10. Relación meta del programa y entregable. Fuente: Elaboración propia 


\section{RESULTADOS}

El análisis inició con la definición de las necesidades de los participantes. Para tal fin, se realizaron diálogos, en los cuales los estudiantes expresaron su percepción respecto al proceso de enseñanza. En la Figura 10 se presenta el resultado del modelo de necesidades. Este modelo permite un acercamiento con la identificación del interesado y sus deseos.

Es de anotar que en proyectos públicos se sugiere que este modelo sea confrontado en varios momentos, considerando los resultados parciales, los supuestos y las restricciones definidas.
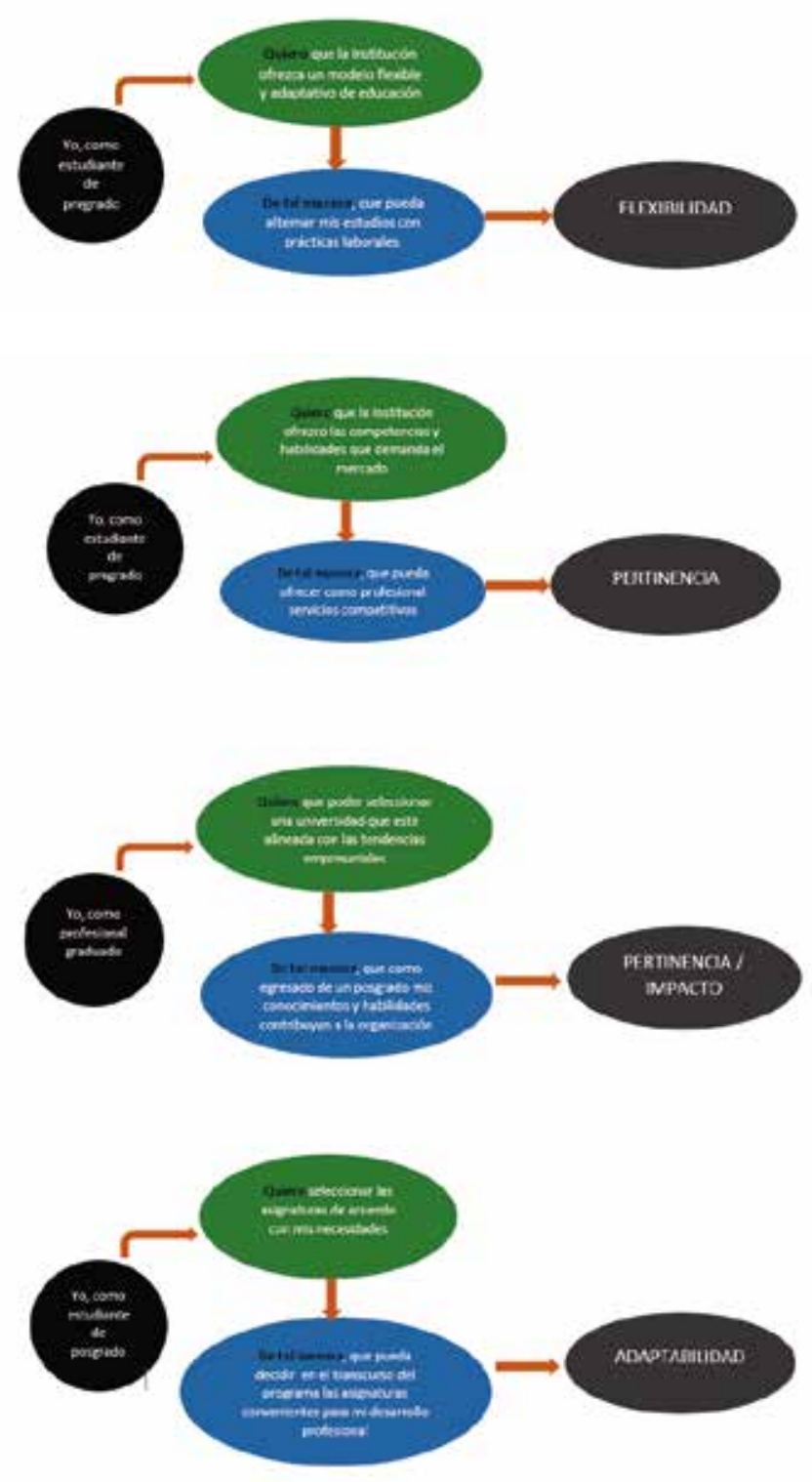

Figura 11. Modelo de gestión de necesidades de estudiantes. Fuente: Elaboración propia
Del análisis de necesidades, se desprende que los estudiantes necesitan una educación flexible, pertinente y adaptativa. Lo cual se traduce en las siguientes definiciones:

Flexible: Un modelo que permite que los estudiantes a medida que avanzan en sus estudios pueden seleccionar las asignaturas que requieren para su desarrollo profesional. En corolario con lo anterior, el tiempo de duración de cada programa debe depender de los objetivos o metas cumplidas.

Pertinente: Programas alineados con las tendencias empresariales, sociales y tecnológicas. En este aspecto, los estudiantes reflejan incomodidad con programas que no se actualizan por lo menos cada dos años. De igual manera, expresan que las Instituciones deben revisar los docentes seleccionados, debido a que éstos no están en el ámbito laboral, por lo tanto, desconocen la dinámica empresarial, y sus requerimientos.

Adaptativa: El modelo de aprendizaje debe apoyarse en la inteligencia artificial y en la ciencia de datos, para ofrecer rutas personalizados de desarrollo académico para cada estudiante de acuerdos con sus resultados. En este sentido, las Instituciones deben avanzar hacia un modelo abierto, en el cual el estudiante pueda seleccionar las asignaturas que crea conveniente para su desarrollo profesional y personal.

En la Figura 12 se relacionan las acciones que están desarrollando los estudiantes para subsanar, así sea parcialmente, sus necesidades de desarrollo académico.

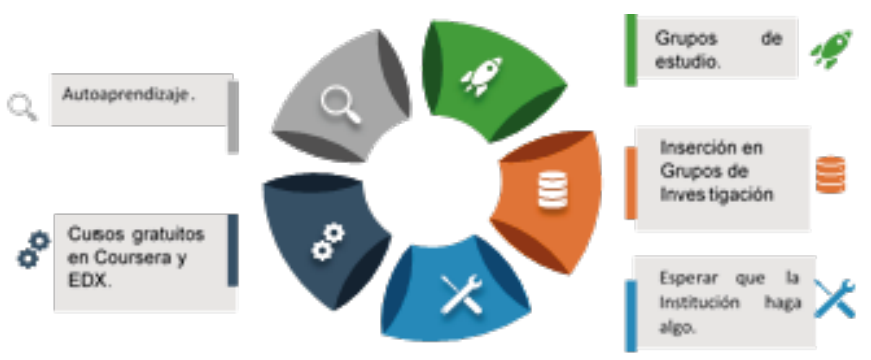

Figura 12. Acciones de los estudiantes. Fuente: Elaboración propia 
En la Figura 13 se consolidan los resultados del trabajo con los grupos de valor a través del Lean Canvas. A partir de este trabajo colaborativo se pueden colegir los siguientes elementos:

- Las Instituciones de Educación Superior, IES, deben diseñar y construir experiencias digitales centralizadas y personalizadas.

- Las IES deben certificarse en la ISO 21001, como medio para garantizar el aseguramiento de los procesos administrativos y académicos. De acuerdo con la percepción de los estudiantes, la Acreditación de Alta Calidad dada por el Ministerio de Educación no asegura pertinencia, flexibilidad y calidad.

- Las IES deben reestructurar la duración de sus programas de acuerdo con el desempeño académico de los estudiantes. Esto quiere decir, que, es a discreción de éstos la elección y el momento de cursar una asignatura.

- Un aspecto en el cual las IES deben fortalecerse es el concerniente a la selección de los docentes. Los estudiantes reportan que éstos están desconectados del ámbito profesional, por lo tanto, desconocen las competencias que demandan las organizaciones.

- La Dirección de Egresados de las IES desempeña un papel fundamental en la definición de las competencias que demanda el sector empresarial. En este sentido, la medición de impacto profesional debe liderarse desde esta dependencia. Además, se sugiere que la definición del grupo factual y contrafactual se realice por Facultad, para potenciar el poder estadístico de la aleatorización.

- La Gestión de datos es una característica de las organizaciones de la Cuarta Revolución Industrial, y las Universidades deben liderar este proceso. Es así, como los estudiantes perciben que las IES no generan valor a partir de los datos que resultan de la interacción de los procesos misionales. Además, de acuerdo con Clemente y Furtado (2018), en la gestión pública de programas es indispensable la definición de instrumentos que permitan medir el estado de madurez de la organización, a partir de la gestión de la información, como resultado de la gestión del conocimiento.

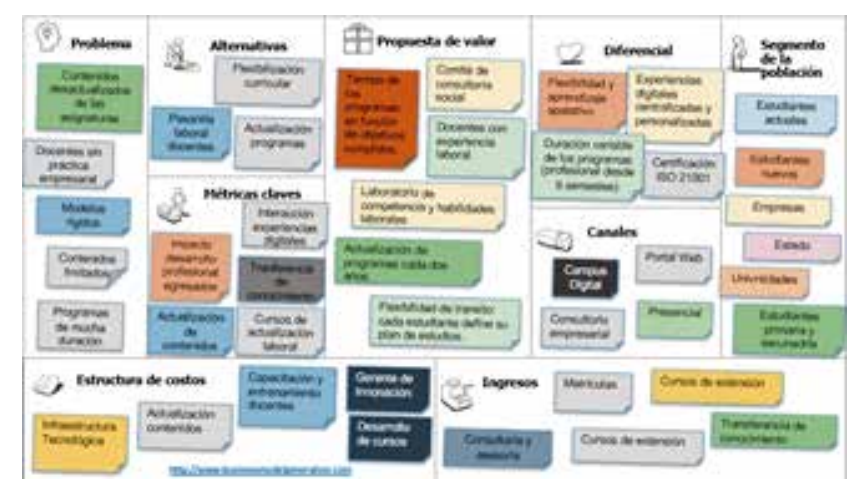

Figura 13. Lean Canvas.

Fuente: Elaboración propia

En la Figura 14 se presenta el modelo de la Teoría del Cambio para la propuesta del programa denominado Educación para el Futuro, cuyo propósito es ofrecer una educación adaptativa, flexible y pertinente, soportada en un modelo robusto de experiencias digitales y analítica de datos. Lo cual permitirá diseñar rutas personalizadas de aprendizaje para cada estudiante.

\section{Componente A - Necesidades e insumos}

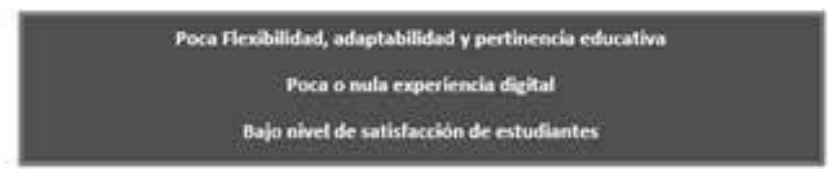

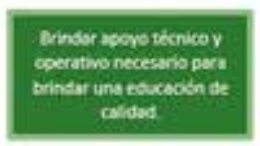

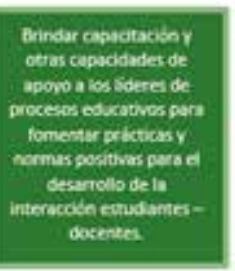

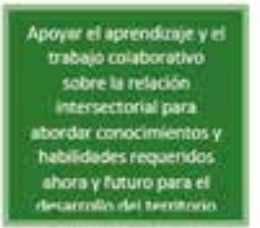

ohop y tuturo pares

Bredecapojo on is

copacidad tikniciy

insinecrevidader de

sctunilixion of

socidartes y eretados.

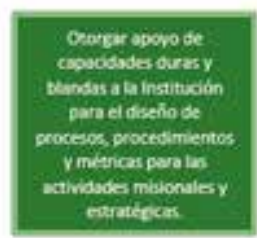

Propoicionscis cormacon ye equpo Gesosidad de grist? institscionat 
Componente B - Acciones
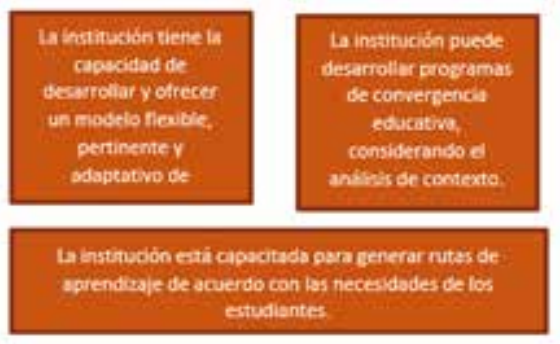

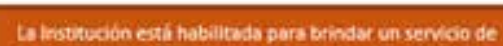

Colidst, a kartir del monitoceo del sistema scademica de coda estudianse

Sefortalecen los capadidodes de la Insatucion parn la sestion y uthilasodon sortenible de los recurvos.

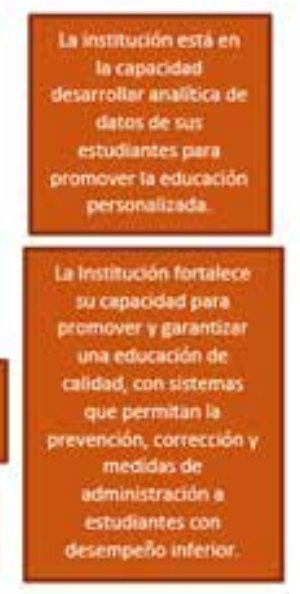

Componente C-Productos
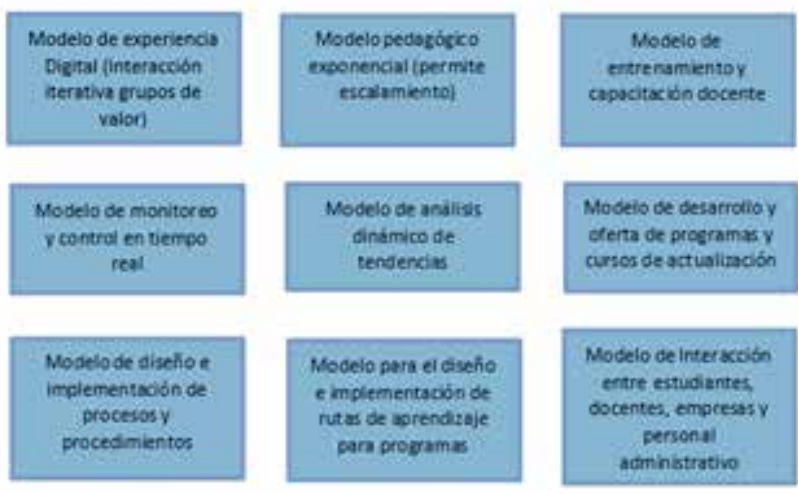

uries de actualiagión
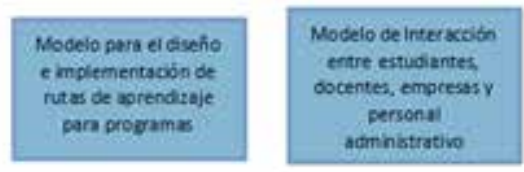

Componente D-

Resultados intermedios y finales
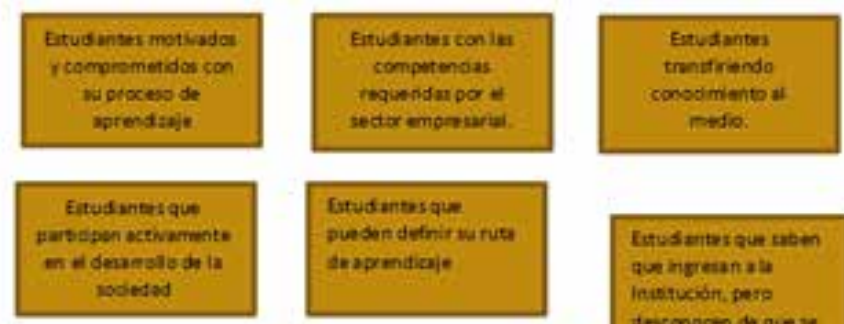

Eitudentes pueden escoge entre uns gras variestas de tunot de actualtacion
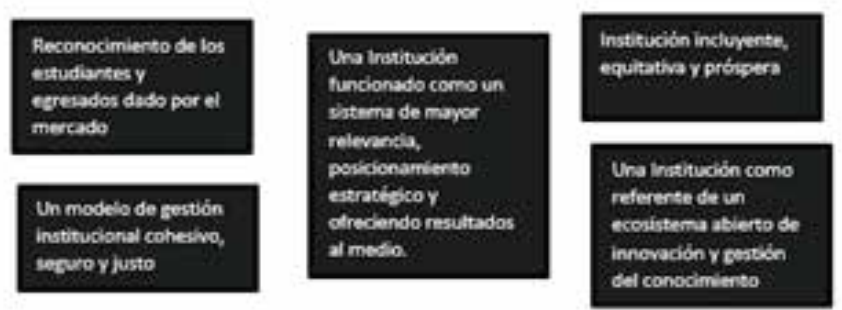

Figura 14. Bloques del modelo de Teoría del Cambio.

Fuente: Elaboración propia
Los supuestos necesarios para el desarrollo de la Teoría del Cambio se enuncian a continuación:

-El proceso actualización educativa es una prioridad para los responsables de la Institución.

- Existe un compromiso por parte de la Institución para fomentar e incrementar el aprendizaje permanente, para mejorar la empleabilidad de los egresados y estudiantes, en consonancia con las necesidades del mercado laboral.

- El Estado aumentará los aportes financieros a las IES en los próximos 3 años.

- La financiación internacional y la asistencia técnica no se reducirán significativamente; será accesible para el diseño e implementación del programa de mejoramiento de la educación.

- Los mecanismos de rendición de cuentas y participación ante el Ministerio de Educación permitirán acceder a recursos financieros, técnicos y de capital humano.

- El Ministerio de Educación moderniza su normatividad, procesos y procedimientos para la oferta, diseño e implementación de programas académicos. Además, flexibiliza el modelo académico.

- Se presentará voluntad política para implementar acciones concernientes para disminuir la tasa de desempleo y reducir la tasa de jóvenes / adultos que no están recibiendo educación y formación.

- Se incrementa la demanda por programa flexibles y adaptativos. Además, se fomentará la investigación aplicada en convenios con el sector empresarial.

- A nivel nacional se desarrollarán leyes para apoyar la formación de programas profesionales de 7 semestres, maestrías de un año y doctorados de 2 años. 
- La analítica de datos permitirá personalizar rutas de aprendizajes de los estudiantes de acuerdo con las exigencias del mercado laboral.

- Los rectores y directivos de las Instituciones serán definidos por un proceso meritocrático.

Considerando los bloques del modelo de Teoría del Cambio, se formulan las siguientes métricas de primer nivel, las cuales luego se desglosan por la Entidad Territorial para la medición del rendimiento del proyecto. Se sugiere la aplicación del modelo Earned Value Management (EVM) para el control de las tres líneas base, alcance, cronograma y costos.

Indicadores de procesos. Estos indicadores consideran el análisis SIPOC, y a partir de esta estructura se definen tasas de utilización de recursos para el logro de los productos del proceso académico y administrativo de la Institución. Por ejemplo:

- Tasa de utilización de la infraestructura tecnológica para labores de docencia, investigación y extensión.

- Tasa de apropiación de la investigación en el aula.

- Tasa de satisfacción de proveedores de infraestructura tecnológica y física.

- Tasa de práctica laboral para docentes.

- Tasa de capacitación y entrenamiento de personal docente y administrativo de la Institución.
Indicadores de insumos. Estos indicadores establecen la relación entre la cantidad de recursos y los resultados de la Teoría del Cambio. Por ejemplo:

- Recursos financieros que requiere la Institución para garantizar educación de calidad por estudiante, de pregrado y posgrado, por semestre. De igual manera, se debe realizar para los cursos de extensión.

- Recursos necesarios para la operación y mantenimiento de la infraestructura tecnológica y física de la Institución.

- Docentes requeridos para garantizar el adecuado proceso de transferencia de conocimiento.

- Tasa de utilización de recursos físicos, financieros y humanos por cada 100 estudiantes. En el análisis de los recursos humanos se hace la diferenciación entre personal administrativo y docentes.

- Tasa de utilización de los contenidos desarrollados para cada una de las asignaturas.

Indicadores de gestión del conocimiento. Estas métricas direccionan la operación del Sistema de Información Gerencial de los Programas. Por ejemplo:

- Tasa de transferencia de los resultados de la investigación a los contenidos de las asignaturas o módulos.

- Tasa de apropiación de las lecciones aprendidas del programa.

- Tasa de transformación de conocimiento tácito a conocimiento explícito en la Institución. Este indicador fomenta la institucionalidad.

- Tasa de Interacción Digital en la plataforma definida para las granjas de conocimiento.

- Tasa de apropiación del proceso de Gestión de la Información y del conocimiento. 
Indicadores de entorno. Estos indicadores relacionan la Institución con el entorno para el apalancamiento del capital social. Por ejemplo:

- Tasa de transferencia de conocimiento al sector empresarial.

- Impacto del modelo educativo en el desarrollo laboral de los egresados (Indicador de largo plazo)

- Tasa de acompañamiento a proyectos sociales. La institución fomenta el desarrollo integral del territorio.

- Tasa de apalancamiento a proyectos de encadenamiento productivo.

- Tasa de capacitación y entrenamiento a la comunidad. Estas capacitaciones se asocian con los análisis de cadena de valor del territorio.

Es frecuente que a partir de la implementación de una política pública resulte un portafolio de programas y proyectos. Por lo tanto, es palmaria la necesidad de un modelo de gestión integral, el cual direccione el diseño e implementación de los proyectos. En este sentido, en la Figura 14 se presenta la estructura del modelo, el cual está constituido por cinco etapas, a saber: visión, análisis, estrategia existente, aprendizaje y estrategia adaptativa. El propósito es la integración de las necesidades, objetivos, métodos e instrumentos, recursos, restricciones y gestión del conocimiento.

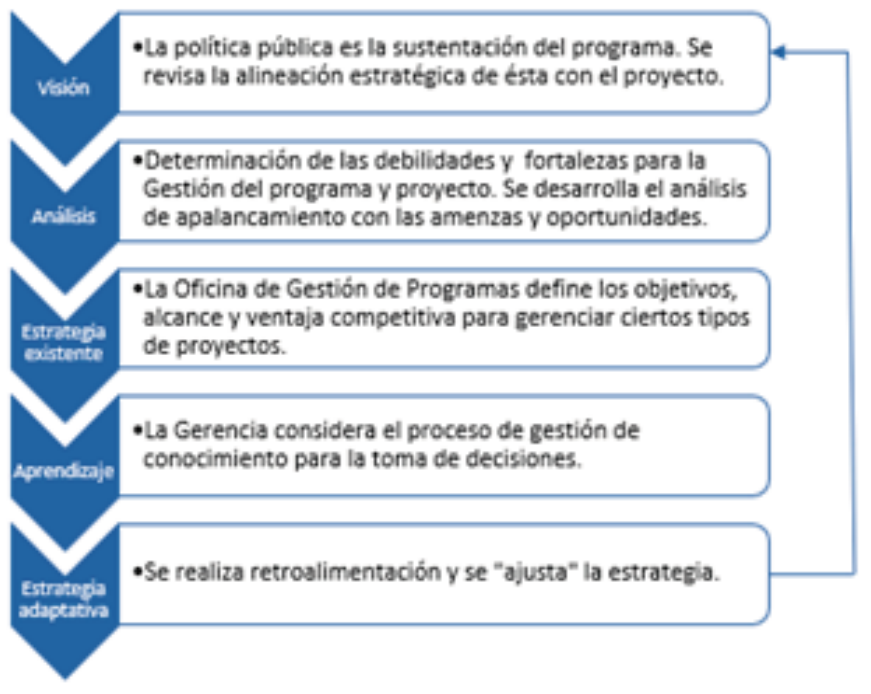

Figura 14. Gestión Estratégica del Programa. Fuente: Elaboración propia

Para el proceso de implementación del modelo de la Teoría del Cambio, se elaboraron 22 preguntas que ayudan a los gestores de programas a desarrollar su etapa de aprestamiento. En este orden de ideas, se debe constatar cada respuesta con su respectiva evidencia.

\begin{tabular}{|c|c|c|c|c|}
\hline Ítem & Acción & Si & No & Observaciones \\
\hline 1 & $\begin{array}{l}\text { ¿Están los objetivos claramente } \\
\text { definidos? }\end{array}$ & & & \\
\hline 2 & $\begin{array}{l}\text { ¿Es claro el alcance del } \\
\text { proyecto para los distintos } \\
\text { grupos de interés? }\end{array}$ & & & \\
\hline 3 & $\begin{array}{l}\text { ¿Los objetivos guardan relación } \\
\text { con el alcance del proyecto? }\end{array}$ & & & \\
\hline 4 & $\begin{array}{l}\text { ¿Las metodologías de los } \\
\text { proyectos están estructuradas y } \\
\text { alineadas con la metodología } \\
\text { general del programa? }\end{array}$ & & & \\
\hline 5 & $\begin{array}{l}\text { ¿Se puede lograr el alcance con } \\
\text { los recursos disponibles y la } \\
\text { metodología propuesta? }\end{array}$ & & & \\
\hline 6 & $\begin{array}{l}\text { ¿Se realiza un uso adecuado de } \\
\text { las fuentes de información? }\end{array}$ & & & \\
\hline 7 & $\begin{array}{l}\text { ¿Los supuestos presentados en } \\
\text { los estudios están claramente } \\
\text { soportados o referenciados? }\end{array}$ & & & \\
\hline 8 & $\begin{array}{l}\text { ¿Se tiene un plan de } \\
\text { comunicaciones claro y } \\
\text { concreto para todo el proyecto? }\end{array}$ & & & \\
\hline 9 & $\begin{array}{l}\text { ¿Se tiene una política clara para } \\
\text { la contratación del capital } \\
\text { humano? }\end{array}$ & & & \\
\hline 10 & $\begin{array}{l}\text { ¿Se tienen definidos } \\
\text { explícitamente las rutas críticas } \\
\text { del proyecto en cada una de sus } \\
\text { áreas? }\end{array}$ & & & \\
\hline 11 & $\begin{array}{l}\text { ¿Se tiene una política de } \\
\text { manejo de la información para } \\
\text { el proyecto? Trazabilidad de } \\
\text { información. }\end{array}$ & & & \\
\hline 12 & $\begin{array}{l}\text { ¿Se tienen establecidos } \\
\text { indicadores de satisfacción de } \\
\text { quejas de la comunidad? }\end{array}$ & & & \\
\hline 13 & $\begin{array}{l}\text { ¿Se tienen directrices definidas } \\
\text { para la viabilidad de programas } \\
\text { y proyectos sociales? }\end{array}$ & & & \\
\hline 14 & $\begin{array}{l}\text { ¿Están los supuestos y } \\
\text { restricciones definidos para } \\
\text { cada uno de los elementos de la } \\
\text { Teoría del Cambio? }\end{array}$ & & & \\
\hline 15 & $\begin{array}{l}\text { ¿La información generada en el } \\
\text { proyecto es transmitida y } \\
\text { comunicada entre todos los } \\
\text { profesionales del proyecto? }\end{array}$ & & & \\
\hline 16 & $\begin{array}{l}\text { ¿Se tiene una matriz de } \\
\text { métricas para el seguimiento al } \\
\text { cumplimiento de los productos, } \\
\text { resultados intermedios y } \\
\text { finales? }\end{array}$ & & & \\
\hline 17 & $\begin{array}{l}\text { ¿En la construcción del modelo } \\
\text { de la Teoría del Cambio se } \\
\text { participó a los interesados del } \\
\text { programa? }\end{array}$ & & & \\
\hline
\end{tabular}




\begin{tabular}{|c|l|l|l|l|}
\hline$\nabla$ & $\begin{array}{l}\text { ¿Existe claridad de la relación } \\
\text { del programa o proyecto con } \\
\text { una política pública? }\end{array}$ & & & \\
\hline 19 & $\begin{array}{l}\text { ¿Se tiene la matriz de gestión } \\
\text { de riesgos, con su análisis de } \\
\text { probabilidad - impacto? }\end{array}$ & & & \\
\hline \multirow{2}{*}{$\begin{array}{l}\text { ¿Se establece un proceso para el } \\
\text { seguimiento de los supuestos, } \\
\text { restricciones, externalidades y } \\
\text { riesgos que considere la cadena } \\
\text { de valor del programa? }\end{array}$} & $\begin{array}{l}\text { ¿Se tiene claridad del proceso } \\
\text { de definición de necesidades de } \\
\text { los grupos de valor? }\end{array}$ & & & \\
\hline 21 & $\begin{array}{l}\text { ¿Se tiene el lean Canvas u otro } \\
\text { modelo para asociar la } \\
\text { propuesta de valor esperada del } \\
\text { programa? }\end{array}$ & & & \\
\hline 22 & & & \\
\hline
\end{tabular}

En la Figura 15 se presenta la propuesta la Oficina de Gestión de Programas, PMO. Ésta integra los Servicios Compartidos con las áreas de la Entidad Pública. Además, mejora los niveles de ejecución estatal. Al momento de su instauración es menester realizar un proceso de capacitación y entrenamiento. Esta propuesta fomenta una visión global y estratégica en la Entidad Pública..

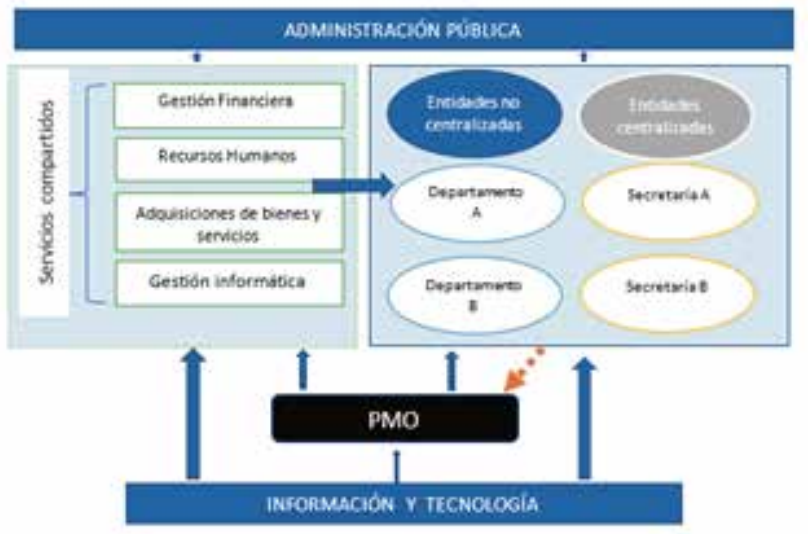

Figura 15. Propuesta de Oficina de Gestión de Programas. Fuente: Elaboración propia

\section{CONCLUSIONES}

Teoría del cambio

La Teoría del Cambio permite el relacionamiento de una necesidad del territorio, con sus productos, resultados e impactos. Además, se convierte en el mapeo del proyecto, lo cual facilita la comunicación con los grupos de valor.

A partir de la Teoría del Cambio es plausible alinear y materializar políticas públicas. Es decir, a través de la relación causal, la Entidad determina sí esta política cumple con su propósito.
La Teoría del Cambio como herramienta de comunicación y gestión de los grupos de valor permite la trazabilidad de los criterios de aprobación de los requerimientos / entregables definidos para el proyecto.

El análisis de supuestos y restricciones es dinámico y debe reflejarse en la matriz de gestión de riesgos. Asimismo, la Entidad gestora debe asegurar que la Teoría del Cambio esté acompañada del análisis de esta matriz. Por otra parte, en el caso de realizar variaciones asociadas a las restricciones, la solicitud de cambio presentada ante Comité Integrado debe reflejar el análisis del impacto de esta restricción sobre las demás.

La presentación de un cambio concerniente a una restricción del programa o proyecto debe surtir las siguientes etapas:

- Evaluar Impacto

- Desarrollar alternativas u opciones

- Revisión interna.

-Validación con el cliente.

Para la implementación de modelo de la Teoría del Cambio es importante que la Entidad diseñe un Sistema de Autorización de Trabajo, el cual evita la corrupción del alcance del programa.

El modelo de la Teoría del Cambio se desarrolla paralelamente con la matriz de gestión de riesgos del programa y proyecto. Del análisis cualitativo se desprenden la lista de priorización de riesgos y la lista de supervisión. La primera se conduce al análisis cuantitativo para la estimación del valor monetario esperado, el cual se refleja en la línea base del proyecto.

Para la definición de la priorización de las variables del programa o proyecto se sugiere el diseño e implementación de la matriz de Vester, a razón de su análisis causal.

Para la medición del impacto en los programas públicos es necesario la definición del factual y el contractual. Esto permite evaluar realmente el efecto de la implementación de una política pública, además, del escalamiento del programa. 
Los estudiantes perciben el Ministerio de Educación como una entidad anquilosada en el pasado, con una comprensión miope de la evolución del desarrollo de los modelos educativos y, por tanto, de la imbricación de la oferta y demanda de competencias.

Las IES aún no satisfacen las necesidades de flexibilidad, pertinencia y adaptabilidad. Estas instituciones soslayan la necesidad de un cambio estructural en sus modelos administrativos y académicos.

Los estudiantes encuentran más apropiación de competencias en plataformas de aprendizaje como Edx, Coursera, Future Learn, entre otros.

Los docentes están desconectados del ámbito laboral. Los estudiantes perciben que, en los diálogos sostenidos en el aula, la retroalimentación del docente es incipiente y desmotiva la asistencia y participación en clase.

Actualmente, los estudiantes perciben que no existe un modelo realmente virtual; más bien, un modelo presencial apoyado en tecnologías de información y comunicación.

Para colegir, la Teoría del Cambio permite revisar la coherencia y pertinencia de una política pública, considerando las necesidades del territorio. 


\section{REFERENCIAS}

Amundsen, C., \& D'Amico, L. (2019). Using Theory of Change to evaluate socially-situated, inquiry-based academic professional development. Studies in Educational Evaluation, 61, 196-208.

Arensman, B., Van Waegeningh, C., \& Van Wessel, M. (2018). Twinning "practices of change" with "theory of change" room for emergence in advocacy evaluation. American Journal of Evaluation, 39(2), 221-236.

Clemente, S. y Furtado, F. (2018). Uma Proposta de Gestão de Projetos para uma Universidade Pública. Gestão.Org, 16, 264-278

DuBow, W. M., \& Litzler, E. (2019). The development and use of a theory of change to align programs and evaluation in a complex, national initiative. American Journal of Evaluation, 40(2), 231-248.

Istrate, L., Marian, L., \& Ferencz, I. S. (2014). The Use of a Project Managenet Application in Managing Investments in a Public Administration Institution. Prodecia Economics and Finance, 15,1732-1739.

PMBOK, G. (2017). Guía de los fundamentos para la dirección de proyectos. Project Management Institute Inc, PMI.

Restrepo, C. \& Cuadros, A. (2013). Evaluación del impacto socioambiental de proyectos. Interciencia, 38(5), 339-346.

Rogers, P. (2014). La teoría del cambio. Síntesis metodológicas: evaluación de impacto, (2), 1-14.

Rogers, P., \& HUMMELBRUNNER, R. (2012). Methodological challenges in using programme theory to evaluate propoor and equity-focused programmes. Evaluation for equitable development results, 142-171.

Stachowiak, S. (2013). Pathways for change: 10 theories to inform advocacy and policy change efforts. Center for Evaluation Innovation. Seattle, WA: ORS Impact.

Sumper, A., Boix-Aragonès, O., Villafáfila-Robles, R., Bergas-Jané, J., \& Ramírez-Pisco, R. (2010). Methodology for the assessment of the impact of existing high voltage lines in urban areas. Energy policy, 38(10), 6036-6044.
Weiss, C. H. (1997). Theory-based evaluation: past, present, and future. New directions for evaluation, 76, 41-55.

Weitzman, B. C., Silver, D., \& Dillman, K. N. (2002). Integrating a comparison group design into a theory of change evaluation: The case of the Urban Health Initiative. American Journal of Evaluation, 23(4), 371-385. 